\title{
Flare and CME Onset: UV Spectra Show Fast 3-D Flow
}

\author{
D. E. Innes \\ Max-Planck-Institut für Aeronomie, D-37191 Katlenburg-Lindau, \\ Germany
}

\begin{abstract}
We present observations taken in the corona above a flare that occurred on the west limb of the Sun. SUMER spectra show large red $\left(350 \mathrm{~km} \mathrm{~s}^{-1}\right)$ and blue $\left(650 \mathrm{~km} \mathrm{~s}^{-1}\right)$ Dopplershifts in Fe XX $\left(10^{7} \mathrm{~K}\right), \mathrm{Cr}$ XVI $\left(5 \times 10^{6} \mathrm{~K}\right)$, Si IX $\left(10^{6} \mathrm{~K}\right)$ and O III $\left(10^{5} \mathrm{~K}\right)$ emission lines. These shifts are associated with a fast moving $\left(400 \mathrm{~km} \mathrm{~s}^{-1}\right)$ optical emission front detected in high cadence images, taken with the coronagraph MICA. SXT images show fast soft X-ray ejecta that can be extrapolated back to the position of pre-flare coronal arcade structure seen in EIT 195 images. The observations are interpreted as evidence of a blast wave propagating through the active region coronal loop structure very early in the flare evolution.
\end{abstract}

Fast moving structures are regularly reported in X-ray, EUV and optical images taken near flare and CME onset (Shibata et al. 1995; Canfield et al. 1996; Shimojo et al. 1996; Nitta \& Akiyama 1999). Because these structures are elongated and/or essentially outwardly moving ejecta many of the scenarios proposed to explain them are based on jet formation and sling-shot ejection flows. In order to gain information on the 3-D flow geometry, a campaign was started to measure Dopplershifts in these jets and ejecta. The SUMER spectrometer was pointed towards the corona above a limb active region. Spectra where obtained every 5 min over a peroid of about 12 hours. In this way, spectra from active region loops before, during and after several flares were obtained. In general, at flare onset there are large Dopplershifts, particularly in the high temperature, $10^{7} \mathrm{~K}$, lines. This is followed by several hours in which the plasma appears to be cooling down with no large-scale Doppler flows.

The event described in this paper is the strongest flare of the campaign and was observed with a unique combination of instruments - SUMER, EIT LASCO and ERNE on SOHO, SXT on Yohkoh and MICA in Argentina. The flare occurred on the west limb of the Sun on May 91999 17:58 UT. The soft X-ray flux began at 17:53 UT and reached maximum, GOES M7.6, at 18:07. The hard X-ray $(50$ - $400 \mathrm{keV})$ light curve showed a single spike lasting $5 \mathrm{~min}$ with a maximum at 17:57:40 UT. A large partial halo CME was seen at 18:27.

A summary of the observations to be discussed here is presented in Fig. 1. The EIT images show the destruction of the $10^{6} \mathrm{~K}$ loop structure. Pre-flare a bright elongated loop-like structure, stretching high into the corona, overlaps the bright top of a lower lying arcade of loops. Together they form a V-like emission pattern above the limb. First, as seen in the middle EIT picture (18:00:11), the tall coronal loop erupted. The lower lying arcade top was still in its original 
position at this time. In the next EIT image, taken 12 min later, the arcade top emission has also disappeared.

In the images on the bottom left of Fig. 1, the optical coronagraph data from MICA have been inset in the 18:00:11 EIT image. The first image has been taken about $30 \mathrm{~s}$ after the max in the hard X-ray emission. There is an optical emission front emerging into the dark part of the corona just north of the bright EIT emission. The front plane-of-sky speed is $400 \mathrm{~km} \mathrm{~s}^{-1}$. The MICA data inset in the forth image (labelled 18:00:12) is the difference between the emission at 18:00:12 and 17:59:29. In this picture the front seems to extend into the brighter loop structures. The outer edge of the front coincides with the top of erupting EIT loop. The optical front emission is predominantly scattered photospheric light which is proportional to the line-of-sight electron column density. Thus the moving optical front indicates a large scale sweeping up of plasma irrespective of temperature, such as expected for a shock wave travelling through the corona.

The two SUMER spectra were taken during the $10 \mathrm{~min}$ around flare onset. The spectrum on the left was taken as the optical front crossed the slit and the EIT loop erupted. It contains lines from O III, Mg IX and $\mathrm{Cr}$ XVI representative of $10^{5}, 10^{6}$ and $5 \times 10^{6} \mathrm{~K}$ gas respectively. There are large Dopplershifts in all lines and at all positions overlapping with the erupting loop and the optical emission front. There is almost no spatial overlap between the enhanced emission in the three temperature regimes, suggesting disruption of a highly structured coronal loop system. Both blue and red shifts are seen. This is particularly clear for the O III line. This emission is coming from the section of the optical front north of the EIT loops. The Dopplershifts, along the slit, show the change in line-of-sight velocity in the optical front (MICA) as new sections of the front cross the slit. The shifts can be interpreted as an expanding loop as first one leg, then the top, then the other leg crosses the slit. The redshift in the $\mathrm{Mg}$ IX $\left(10^{6} \mathrm{~K}\right)$ line and the Cr XVI $\left(5 \times 10^{6} \mathrm{~K}\right)$ line are coming from different initial loop structures, that have been ejected in opposite directions. The second spectrum containes lines from Si IX and Fe XX representative of $8 \times 10^{5}$ and $10^{7} \mathrm{~K}$ gas. Oppositely directed hot plasma flow is seen in the Fe XX line, at exactly the position where the soft X-ray difference image (lower right panel) shows expansion of hot gas northwards along the UV spectrometer slit. At the same time, hot X-ray ejecta are moving outwards with a velocity of $550 \mathrm{~km} \mathrm{~s}^{-1}$ from the arcade top into the corona. Extrapolating back in time, this ejecta would have left arcade top at 18:00:30 (i.e. after the optical front had passed).

The most strightforward interpretation of the observed large-scale, fastmoving optical front, the associated loop disruption, the red and blue Doppler shifts and high temperature plasmoid ejection is the interaction of a blast wave with a highly structured coronal loop system.

\section{References}

Canfield, R. C. et al. 1996, ApJ, 464, 1016

Nitta, N., \& Akiyama, S. 1999, ApJ, 525, L57

Shibata, K. et al. 1995, ApJ, 451, L83

Shimojo, M. et al. 1996, PASJ, 48, 123 
A. EIT

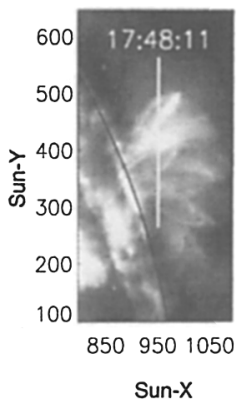

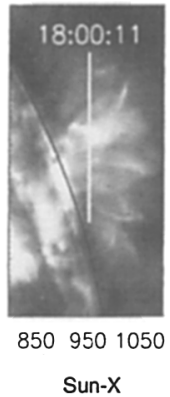

Sun-X

\section{B. SUMER}
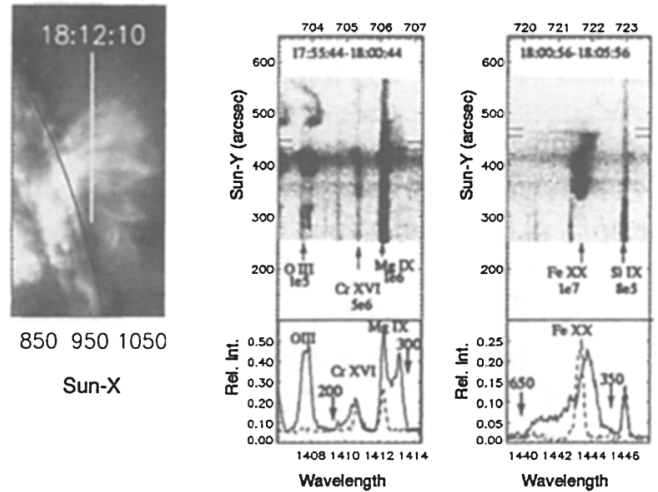

C. MICA and EIT
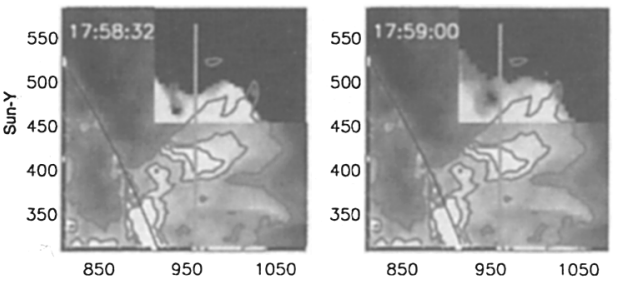

D. SXT and EIT
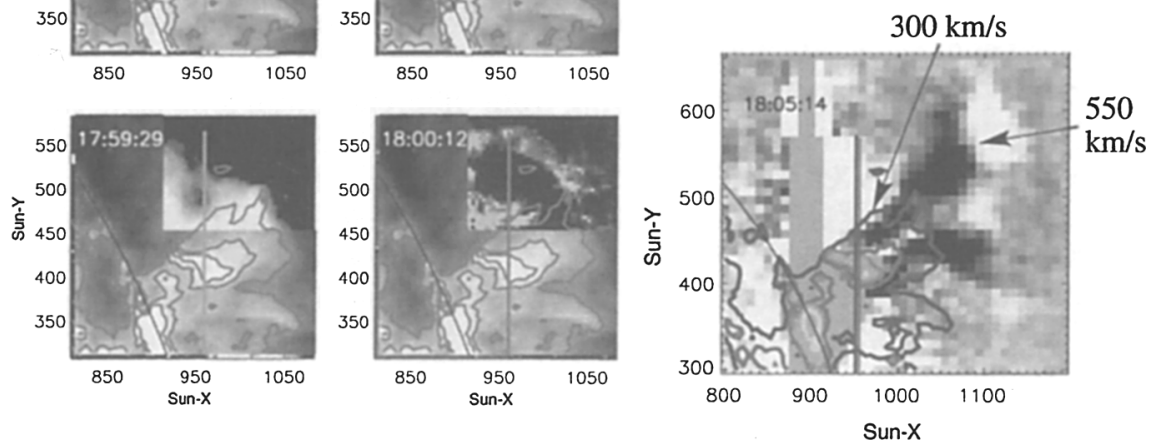

Figure 1. Association between structures seen by different instruments at flare onset. The flare occurred on the west limb. The position of the SUMER slit is shown as a vertical line in EIT, MICA and SXT images. The line spectra in the SUMER panels are the integrated emission along the section of the slit marked by extended ticks on the image above and the dashed line shows the unshifted profile from a spectrum taken 3.5 hours later. 